\title{
DETECÇÃO DE FÓTONS ISOLADOS EM 1550 NM: SIMULAÇÃO E RESULTADOS EXPERIMENTAIS
}

\author{
George André Pereira Thé e Rubens Viana Ramos
}

\begin{abstract}
Resumo - Distribuiçào quântica de chaves é uma técnica que permite a distribuição segura de uma string de bits que é usada como chave em protocolos criptográficos. A segurança da distribuição é garantida por uma adequada codificação da informação em uma propriedade quântica, e pela indivisibilidade e impossibilidade de clonar um quantum. Assim, para a implementação prática de redes ópticas capazes de suportar protocolos quanto-criptográficos, é necessária a construção de detectores capazes de detectar um fóton. Neste artigo são apresentados simulações numéricas e resultados experimentais de um detector de fótons isolados, usando fotodiodos de avalanche, na janela de $1550 \mathrm{~nm}$.
\end{abstract}

Palavras-chave: Detectores de fótons isolados, fotodiodo de Avalanche, distribuição quântica de chaves.

Abstract - Quantum key distribution is a new technique that permits secure distribution of a bit string, used as key in cryptographic protocols. The security is guaranteed by suitable coding of the information in a quantum property and from the fact that a quantum cannot be both split and cloned. The present quantum-cryptographic protocols use a quantum (photon in optical systems) to carry the information. Hence, for the construction of an optical set up able to run quantum-cryptographic protocols, it is necessary use single-photon detectors. In this paper, numerical simulations and experimental results of single-photon detectors, in $1550 \mathrm{~nm}$, using avalanche photodiode are shown.

Keywords: Single-photon detectors, avalanche photodiode, quantum key distribution.

\section{INTRODUÇÃO}

Distribuição quântica de chaves (DQC) é uma técnica recente que permite a troca segura de uma string de bits, usada como chave em um algorítmo de criptografia [1,2]. A segurança dos protocolos é garantida por três fatores: uma adequada codificação da informação em uma propriedade quântica (por exemplo, a fase ou a polarização da luz), a indivisibilidade de um quantum e a impossibilidade de clonar um estado quântico [3]. Para a implementação de protótipos capazes de suportar protocolos de DQC é indispensável a construção de bons detectores de fótons isolados. Para este fim, fotodiodos de avalanche (FDA) são usados. Como os FDAs comercialmente disponiveis não são construídos para detecção de fótons isolados na janela de $1550 \mathrm{~nm}$, torna-se importante a caracterização dos mesmos na realização de tal função. Para este fim descrevemos, na Seção 2 os

George André Pereira Thé e Rubens Viana Ramos estão na Universidade Federal do Ceará, Departamento de Engenharia de Teleinformática, Campus do Pici, Fortaleza, CE, Brasil. E-mails: \{geothe, rubens\}@deti.ufc.br. Editor de Área responsável: Denise Consonni. Artigo submetido em 01/Ago/2002, revisado em $01 /$ Nov/2002, aceito em 19/Set/2003. principais parâmetros do FDA, nas Seções 3 e 4 o funcionamento do circuito de extinção gatilhado e, na Seção 5, a caracterização experimental do FDA.

\section{FUNCIONAMENTO E PARÂMETROS DO FDA}

Basicamente, um receptor óptico em sistemas de DQC deve receber um fóton em sua entrada e gerar um pulso elétrico em sua saída. Como o nível de potência óptica devido a um fóton nas janelas de comunicações ópticas é muito baixo, o receptor não deve introduzir ruído sob pena de não conseguir distinguí-lo do sinal de informação. Entretanto, um receptor óptico capaz de receber um fóton e gerar uma corrente elétrica possível de ser processada deve, necessariamente, usar um fotodiodo de avalanche (FDA). Esses dispositivos são intrinsecamente ruidosos e, por isso, algumas precauções devem ser tomadas para viabilizar o uso de FDAs como, por exemplo, o resfriamento dos mesmos. Para a região de $1300 \mathrm{~nm}$ os FDAs de Ge são utilizados, enquanto que na janela de $1550 \mathrm{~nm}$, melhor devido à baixa perda da fibra, FDAs de InGaAs/InP são utilizados. Basicamente o FDA funciona da seguinte forma [4,5]: os fótons que chegam ao FDA são absorvidos na camada intrínseca do mesmo; cada fóton que é absorvido pode gerar um par elétron-lacuna; os portadores gerados na região intrínseca atravessam a região de ganho acelerados pela tensão de alimentação e podem, dependendo do nivel de tensão aplicado ao FDA, ganhar energia suficiente para gerar novos pares elétron-lacuna ao colidir com outros átomos da estrutura, dando início a um processo de avalanche. O processo de avalanche começa a ocorrer a uma dada polarização reversa aplicada ao FDA, chamada tensão de ruptura (breakdown voltage). A quantidade de tensão aplicada ao FDA acima da tensão de ruptura é chamada tensão de excesso. Os principais parâmetros do FDA na construção de detectores de 1-fóton são: eficiência quântica, probabilidade de contagem de escuro e afterpulsing. A eficiência quântica, $\eta$, é resultado de três fatores: 1) a probabilidade do fóton absorvido gerar um par elétron-lacuna; 2) a probabilidade dos portadores gerados dispararem uma avalanche; 3) a eficiência do acoplamento óptico da fibra com o dispositivo semicondutor. A eficiência quântica cresce com o aumento da temperatura e da tensão de excesso, $V_{e}=V_{F D A}-V_{h}$, onde $V_{F D A}$ é a tensão sobre o FDA e $V_{b}$ a tensão de ruptura. A probabilidade de contagem de escuro, $P_{e s c}$, é a contagem devida às avalanches causadas por portadores originados por efeitos térmicos, de tunelamento e armadilhas (trapping). Essas contagens indevidas provocam erros nos sistemas DQC. Assim como $\eta, P_{\text {esc }}$ aumenta com o aumento da temperatura e de $V_{e}$. O afterpulsing é um fator contributivo para contagens indevidas no fotodetector. Após a avalanche cessar alguns portadores podem ficar presos em armadilhas (traps) na região de alto campo onde ocorre a ionização de 
impacto. Após algum tempo (tempo médio de vida dos portadores aprisionados que é inversamente proporcional à temperatura), estes portadores se libertam e podem originar uma nova avalanche. A quantidade de afterpulses é proporcional à carga que flui através do dispositivo durante a avalanche, significando que o afterpulsing cresce com $V_{e}$. Como será visto posteriomente, o afterpulse limita a taxa de transferência de dados do sistema DQC. O NEP (noise equivalent power) é uma figura de mérito que fornece a melhor relação entre sinal e ruído do FDA. Ele é uma função dos vários parâmetros que influem na performance do fotodiodo. A performance será tão melhor quanto menor for o $N E P$. O NEP é calculado pela equação $[6,7]$

$$
N E P=h v\left(2 R_{d}\right)^{1 / 2} / \eta \quad\lfloor W / \sqrt{H z}\rfloor
$$

sendo $R_{d}$ a taxa de contagem de escuro, isto é, número de contagens por unidade de tempo.

\section{CIRCUITOS DE EXTINÇÃO DE AVALANCHE}

Quando o FDA é usado no modo Geiger $\left(V_{F D A}>V_{b}\right)$, após uma avalanche ter sido iniciada ela deve ser extinta para não danificar o fotodiodo. Isto pode ser obtido por um circuito que percebe a avalanche e a extingue reduzindo, momentaneamente, a tensão sobre o diodo para um valor abaixo de $V_{b}$. Este processo pode acontecer a partir de 3 diferentes técnicas: modo passivo, modo ativo e modo gatilhado. Também podem ser usadas combinações destas técnicas. Em sistemas DQC o modo gatilhado é o mais usado e, por isso, vamos nos restringir a ele. O circuito de extinção gatilhado é indicado para os casos em que a chegada dos fótons ocorre segundo uma taxa conhecida. A tensão sobre o diodo é mantida acima de $V_{b}$ somente em janelas de tempo de duração $T_{w}$, durante as quais uma avalanche pode ocorrer. Estes períodos são separados por janelas temporais de desuso (hold-off time) de duração $T_{h}$, nestas $V_{F D A}$ é mantido abaixo de $V_{b}$ e, portanto, a avalanche não pode ocorrer. A Figura 1 ilustra um trem de pulsos de gatilho.

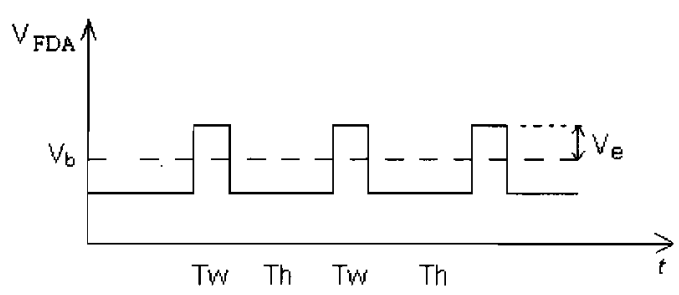

Figura 1. Trem de pulsos de gatilho.

Na Figura 2, o circuito de extinção gatilhado é apresentado $[6,7]$. Nesta figura, $V_{g}$ é a fonte de pulsos de gatilho, $C_{g}$ é o capacitor de acoplamento, $R_{S}$ é o resistor de carga onde o pulso de avalanche é detectado, $R_{/}$limita a corrente que flui pelo FDA e, em circuitos de extinção passivos é o responsável pela extinção da avalanche.

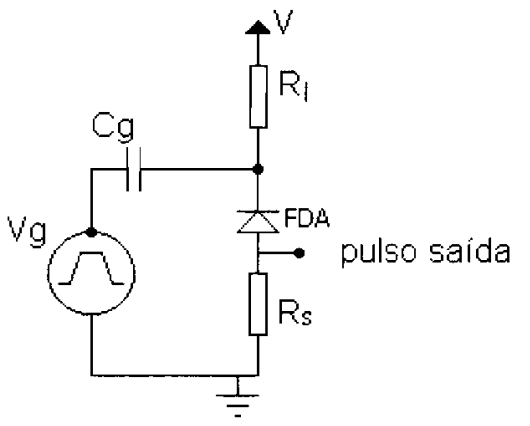

Figura 2. Circuito de extinção gatilhado.

O pulso retangular (Figura 1) aplicado ao fotodiodo é modificado pelo filtro diferenciador feito pelo capacitor $C_{g}$ em série com $R_{l}$ e paralelo com $C_{d}+C_{s}$ (capacitâncias interna do FDA). O pulso que chega ao fotodiodo, $V_{g}{ }^{\prime}$, é dado por

$$
V_{g}^{\prime}=V_{g}\left[C_{g} /\left(C_{g}+C_{d}+C_{s}\right)\right]
$$

Para reduzir a perda de amplitude de $V_{g}$ deve-se usar um alto valor para $C_{g}$. Quando os pulsos de gatilho são muito largos, a extinção ocorre através de $R$. Nestas condições o tempo de extinção é dado por $[4,7]$

$$
T_{g q}=R_{l} R_{d}\left(C_{g}+C_{d}+C_{s}\right) /\left(R_{l}+R_{d}\right)
$$

sendo $R_{d}$ a resistência interna do diodo. O tempo de recueração, isto é, o tempo necessário para o fotodiodo ficar apto a iniciar uma nova avalanche, é dado por $[4,7]$

$$
T_{g r}=R_{l}\left(C_{g}+C_{s}+C_{d}\right)
$$

O circuito de extinção gatilhado tem como princip :antagem a grande redução de contagens de escuro, uni.: 'ez que o FDA só estará "ligado" em curtos intervalos de tempo. Para reduzir o afterpulsing deve-se usar uma baixa freqüência de pulsos de gatilho, de forma que os portadores aprisionados possam se libertar entre dois pulsos de gatilho. Portanto, o afterpulsing limita a taxa de recebimento de fótons limitando, portanto, a velocidade de comunicação.

\section{SIMULAÇÃO DO CIRCUITO DE EXTINÇÃO GATILHADO}

O circuito de extinção gatilhado, Figura 2, é novamente mostrado na Figura 3. Nesta figura vemos que o FDA é representado por sua resistência e capacitância internas, $R_{d}$ e $C_{d}$, respectivamente, e por $C_{s}$ que representa as capacitâncias de fuga. Por fim, a chave $C_{h}$ fica aberta (fechada) na ausência (presença) da avalanche. A tensão $V_{g}^{\prime}$ e a resistência $R$ são dadas por

$$
\begin{gathered}
V_{g}^{\prime}=V_{g}\left(R_{i n} / R_{i n}+R_{g}\right) \\
R=R_{i n} R_{g} /\left(R_{i n}+R_{g}\right)
\end{gathered}
$$

sendo $R_{g}$ a resistência interna da fonte $V_{g}$ e $R_{\text {in }}$ a resistência de entrada colocada entre $C_{g}$ (ponto 1) e o terra, para casar a 
impedância de entrada do circuito de extinção com a impedância de saida da fonte $V_{g}$.

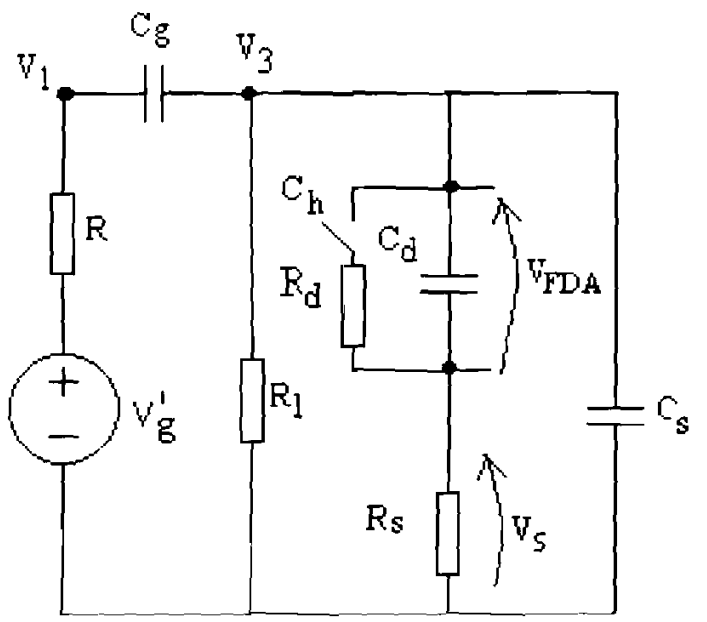

Figura 3. Modelo $a c$ do circuito de extinção gatilhado.

As equaçōes de estado do circuito da Figura 3 são

$$
\begin{aligned}
& \frac{d V_{1}}{d t}+\frac{V_{1}}{R C_{g}}-\frac{d V_{3}}{d t}=\frac{V_{g}^{\prime}}{R C_{g}} \\
& \frac{d V_{F D A}}{d t}+\frac{V_{F D A}}{C_{d} R_{2}}=\frac{V_{3}}{C_{d} R_{s}} \\
& \frac{d V_{3}}{d t}+\frac{V_{3}}{C_{s}\left(R_{l} \| R_{s}\right)}+\frac{V_{1}}{R C_{s}}-\frac{V_{F D A}}{C_{s} R_{s}}=\frac{V_{g}^{\prime}}{R C_{s}}
\end{aligned}
$$

sendo $R_{2}=R_{s}$ quando não há avalanche e $R_{2}=R_{s} \| R_{d}$ durante a avalanche. Discretizando (7)-(9), obtemos

$$
\begin{aligned}
& V_{3}(n+1)=T\left[\begin{array}{l}
\frac{V_{g}^{\prime}(n)}{\tau_{3}}+\left(\frac{1}{T}-\frac{1}{\tau_{2}}\right) V_{3}(n) \\
-\frac{V_{1}(n)}{\tau_{3}}+\frac{V_{F D .4}(n)}{\tau_{4}}
\end{array}\right], \\
& V_{1}(n+1)=T\left[\begin{array}{l}
\frac{V_{g}^{\prime}(n)}{\tau_{1}}+\left(\frac{1}{T}-\frac{1}{\tau_{1}}\right) V_{1}(n) \\
+\frac{V_{3}(n+1)-V_{3}(n)}{\tau_{3}}
\end{array}\right], \\
& V_{F D A}(n+1)=T\left[\frac{V_{3}(n)}{\tau_{5}}+\left(\frac{1}{T}-\frac{1}{\tau_{5}}\right) V_{F D A}(n)\right] \text {, }
\end{aligned}
$$

sendo que as constantes de tempo são $\tau_{1}=R C_{g}, \tau_{2}=C_{s}\left(R_{s}|| R_{l}\right)$, $\tau_{3}=R C_{s}, \tau_{4}=R_{s} C_{s}, \tau_{5}=C_{d}\left[R_{s} R_{d} /\left(k R_{s}+R_{d}\right)\right]$. A variável aleatória $k$ assume o valor 0 quando não há avalanche, e 1 durante uma avalanche. Nas simulações as tensões $V_{1}, V_{F D A} \mathrm{e}$ $V_{s}=V_{3}-V_{F D \text {. }}$ são mostradas. Nas figuras 4-7 vemos, respecti- vamente, para a extinção ocorrendo no fim do pulso de gatilho $\left(T_{m}<T_{g q}\right)$, a seguinte seqüencia de eventos: ausência de avalanche, avalanche ocorrendo no início, no meio e no fim do pulso de gatilho. Os valores dos parâmetros utilizados são: $R_{\mathrm{g}}=R_{\text {in }}=R_{s}=0,050 \mathrm{k} \Omega, R_{d}=1 \mathrm{k} \Omega, R_{f}=330 \mathrm{k} \Omega, C_{\mathrm{g}}=100 \mathrm{pF}$, $C_{d r}=C_{s}=0,5 \mathrm{pF}, V_{\mathrm{g}}=6$ e $T_{\mathrm{u}}=8,4 \mathrm{~ns}$.

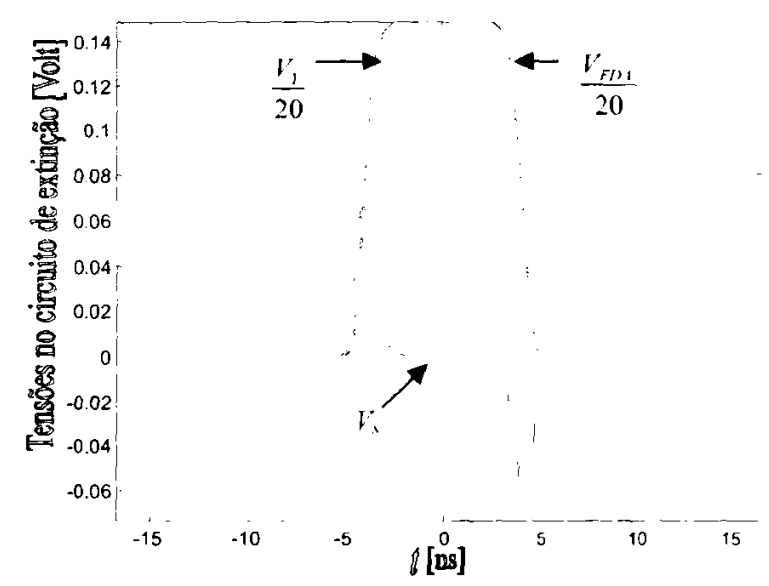

Figura 4. Pulsos de gatilho, $V_{F D A}$ e de saída $\left(T_{w}<T_{g q} \rightarrow\right.$ $R_{l}=330 \mathrm{k} \Omega$ ): ausência de avalanche.

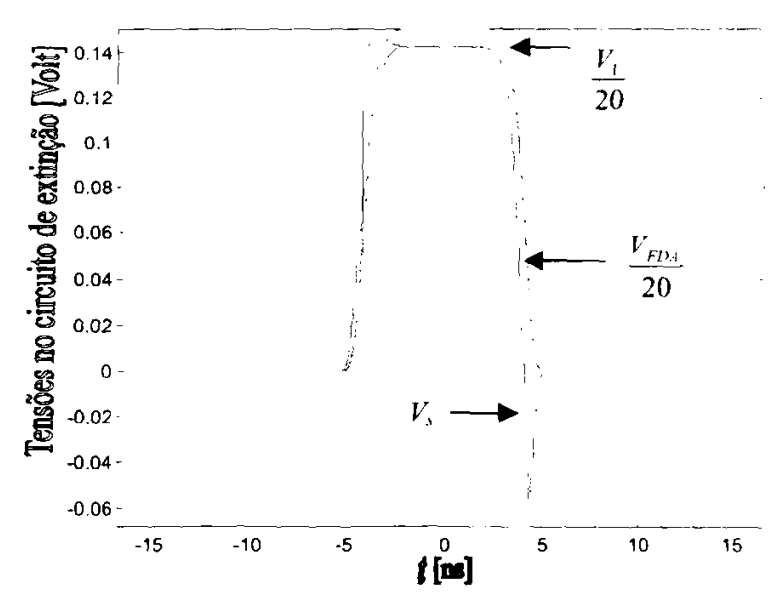

Figura 5. Pulsos de gatilho, $V_{F D A}$ e de saida $\left(T_{n}<T_{R q} \rightarrow\right.$ $R_{I}=330 \mathrm{k} \Omega$ ): avalanche no início do pulso de gatilho.

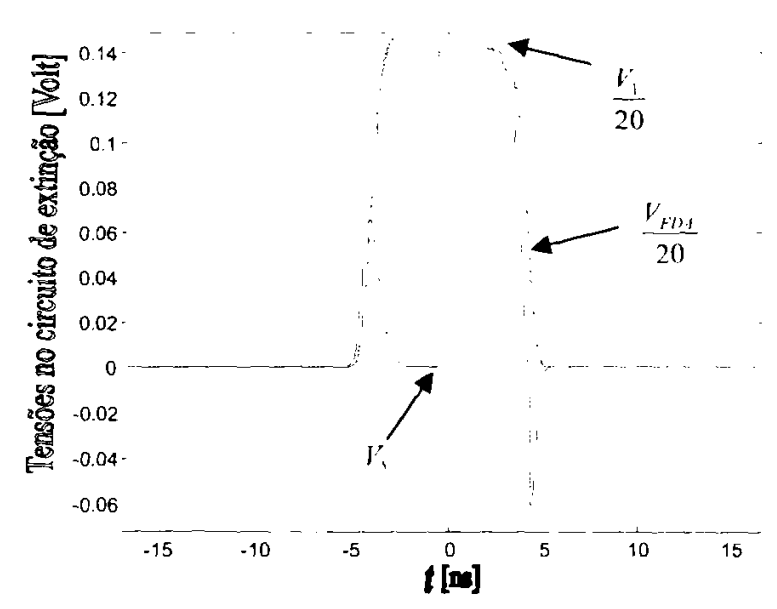

Figura 6. Pulsos de gatilho, $V_{F D A}$ e de saida $\left(T_{11}<T_{g q} \rightarrow\right.$ $R_{l}=330 \mathrm{k} \Omega$ ): avalanche no meio do pulso de gatilho. 


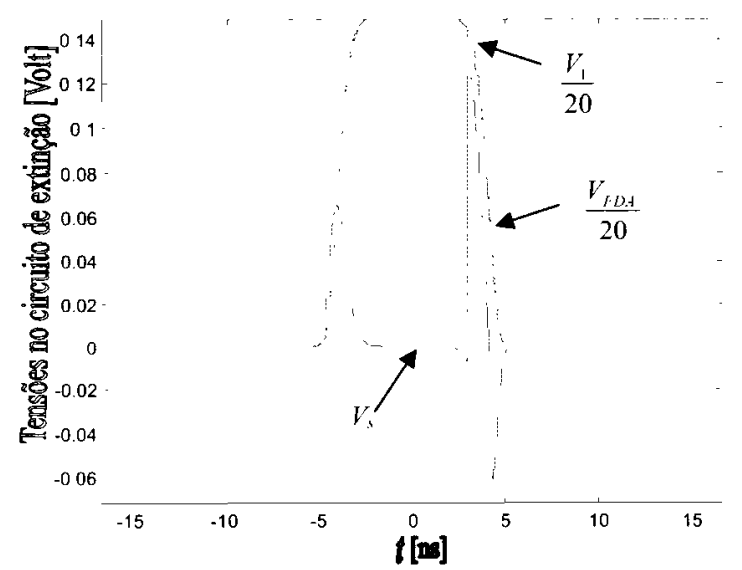

Figura 7. Pulsos de gatilho, $V_{F D A}$ e de saida $\left(T_{n}<T_{g q} \rightarrow\right.$ $R_{l}=330 \mathrm{k} \Omega$ ): avalanche no fim do pulso de gatilho.

Na Figura 4 vemos que, mesmo na ausência de avalanche, há a presença de um estreito pulso devido à resistência das capacitâncias à rápida transição nas bordas do pulso de gatilho. Em estágios posteriores, o nível de comparação deve ser maior que o valor de pico daquele pulso, sob pena de acusar uma falsa contagem. Na Figura 5 temos a melhor situação de detecção uma vez que a avalanche dura todo o intervalo da janela de gatilho. Na Figura 6 temos a situação mais provável e ainda favorável de detecção. Por fim, na Figura 7 vemos que o pulso de avalanche é bastante estreito, exigindo dos estágios posteriores de comparação e amplificação resposta rápida, ou seja, grande largura de banda. As figuras 8 e 9 mostram, respectivmente, para a extinção da avalanche ocorrendo durante o pulso de gatilho $\left(T_{w}>T_{g q}\right)$, a seguinte seqüência de eventos: avalanche ocorrendo no início e no meio do pulso de gatilho. Nestas simulações $R_{l}$ $=0,050 \mathrm{k} \Omega$ e os demais parâmetros têm os mesmos valores usados nas simulações anteriores.

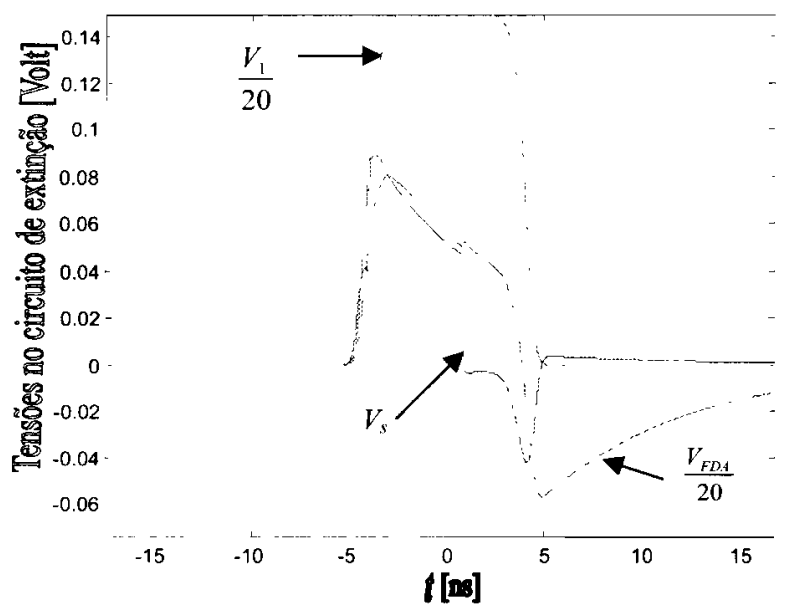

Figura 8. Pulsos de gatilho, $V_{F D A}$ e de saída $\left(T_{n}>T_{g q}\right.$ $\rightarrow R_{l}=50 \Omega$ ): avalanche no início do pulso de gatilho.

Observamos nas figuras 8 e 9 que a tensão sobre o FDA, $V_{F D A}$, decresce continuamente. Isto ocorre devido ao carregamento rápido de $C_{g}$ através de $R_{l}$. Um menor valor de $V_{F D A}$ implica em uma menor probabilidade de uma avalanche ocorrer. Praticamente não ocorre avalanche no fim do pulso de gatilho pois a tensão sobre o fotodiodo já será muito baixa.

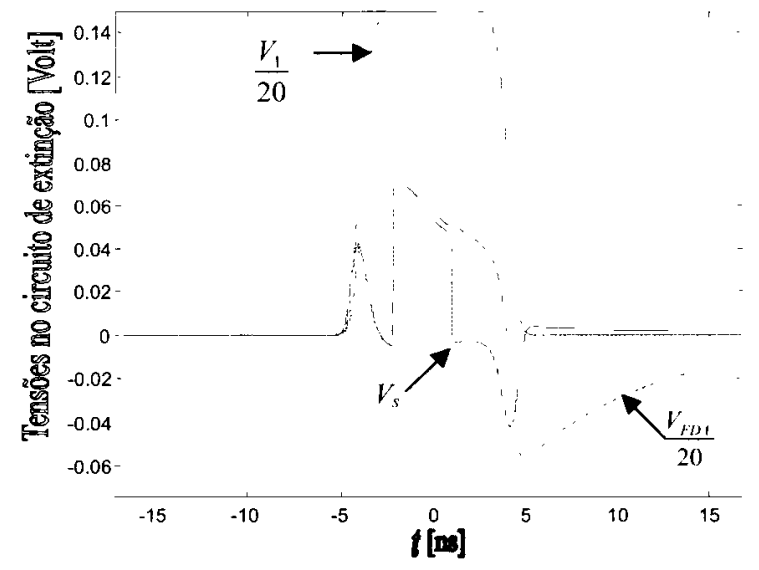

Figura 9. Pulsos de gatilho, $V_{F D A}$ e de saída $\left(T_{n}>T_{g q} \rightarrow\right.$ $R_{l}=50 \Omega$ ): avalanche no meio do pulso de gatilho.

É importante notar que, ao final do pulso de gatilho, $V_{F D A}$ assume um valor de pico negativo e demora um determinado tempo para atingir o valor zero novamente. A chegada de um novo pulso de gatilho antes que haja uma completa recuperação provocará um menor valor de pico da tensão sobre o FDA, como mostra a Figura 10.

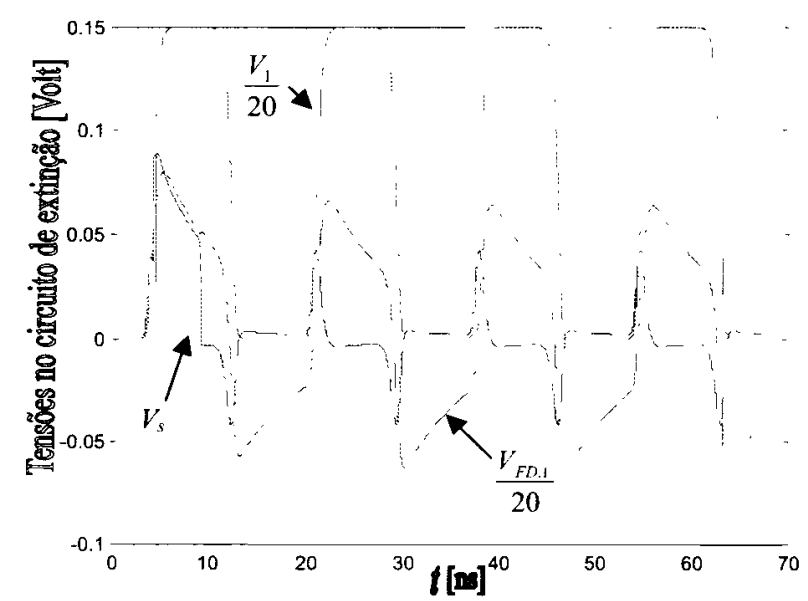

Figura 10. Seqüência de 4 pulsos de gatilho, $V_{F D A}$ e de saída $\left(T_{\mathrm{r}}>T_{g q} \rightarrow R_{l}=50 \Omega\right)$.

Para evitar este efeito, o espaçamento entre dois pulsos de gatilho deve ser maior que o tempo necessáio para a tensão sobre o FDA retornar a zero, o que limita a taxa de transmissão de dados. Na simulação da Figura 10 foram usados os mesmos parâmetros da simulação anterior (figuras 8 e 9 ).

\section{CARACTERIZAÇÃO EXPERIMENTAL DO FDA}

A caracterização do FDA é realizada com o esquema mostrado na Figura 11. Nesta figura podemos observar que: 1) Junto ao FDA está o circuito de extinção, o elemento peltier e o sensor de temperatura (termistor). Estes dois 


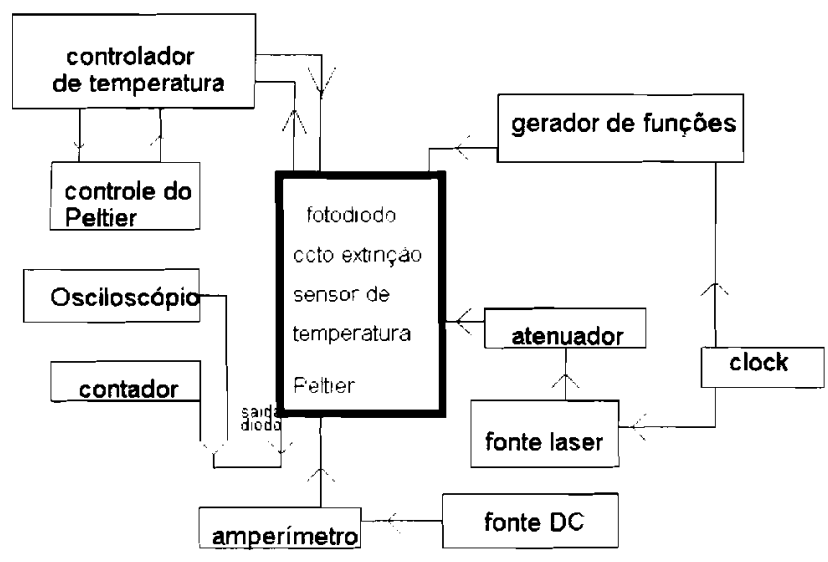

Figura 11. Esquema experimental para caracterização do FDA.

últimos fazem parte do controle de resfriamento do FDA. O resfriamento é necessário para manter a probabilidade de contagem de escuro baixa;

2) À saída do circuito está ligado um osciloscópio (para visualizaçâo da forma e amplitude do pulso de saída) e um contador que contará o número de ava-lanches que são produzidas em um determinado intervalo de tempo;

3) Há um circuito de controle de temperatura;

4) Para simular uma fonte de luz de um fóton a luz gerada por um laser semicondutor DFB é fortemente atenuada para possuir, em média, 0,1 fótons/pulso. Com isso, na maioria das vezes que um pulso alcançar o FDA ele terá apenas um fóton;

5) O gerador de funções fornece os pulsos de gatilhos;

6) Há um sincronismo entre a fonte laser e o gerador de funções, de forma que os pulsos de gatilho e os fótons cheguem ao FDA ao mesmo tempo.

A probabilidade de uma contagem de escuro é $P_{e s c}=R_{e s c} T_{n}$, onde $R_{e s c}$ é a taxa de contagem de escuro, suposta ser constante. Para determinar a probabilidade de contagem de escuro procedemos da seguinte forma: sem a fonte laser conectada, para uma frequeência $f(1 \mathrm{kHz})$ de chegada dos pulsos de gatilho, medimos o número de contagens, $N_{\text {esc }}$, detectadas durante o intervalo $T_{i}=100 \mathrm{~s}$. A probabilidade $P_{e s c}$ é dada por

$$
P_{e s c}=N_{e s c} / T_{i} f .
$$

O resultado experimental da probabilidade de contagem de escuro pode ser observado na Figura 12, para valores de amplitude do pulso de gatilho $\left(V_{g}\right)$ no intervalo $[1 \mathrm{~V}, 5 \mathrm{~V}] \mathrm{e}$ temperaturas no intervalo $\left[-150^{\circ} \mathrm{C},-30^{\circ} \mathrm{C}\right]$. A tensão de alimentação do FDA foi colocada $0,5 \mathrm{~V}$ abaixo da tensão de ruptura do fotodiodo. Como esperado, observamos que quanto maiores forem a temperatura e amplitude de $V_{g}$ maior a probabilidade de contagem de escuro. Para determinar experimentalmente a eficiência quântica do FDA procedemos da seguinte forma: com a fonte laser conectada, para uma freqüência $f(1 \mathrm{kHz})$ de chegada dos pulsos de gatilho, medimos o número de contagens, $N_{l i t}$, detectadas durante um intervalo $T_{i}=100 \mathrm{~s}$. A probabilidade de contagem com laser ligado, $P_{l t z}$, e a eficiência quântica são dados por

$$
\begin{aligned}
& P_{l u=}=\frac{N_{l u z}}{T_{i} f} \\
& \eta=\ln \left[\left(\frac{1-P_{e s c}}{1-P_{l u z}}\right)^{1 /\langle n\rangle}\right]
\end{aligned}
$$

sendo $\langle n\rangle(=0,1)$ o número médio de fótons chegando ao FDA.

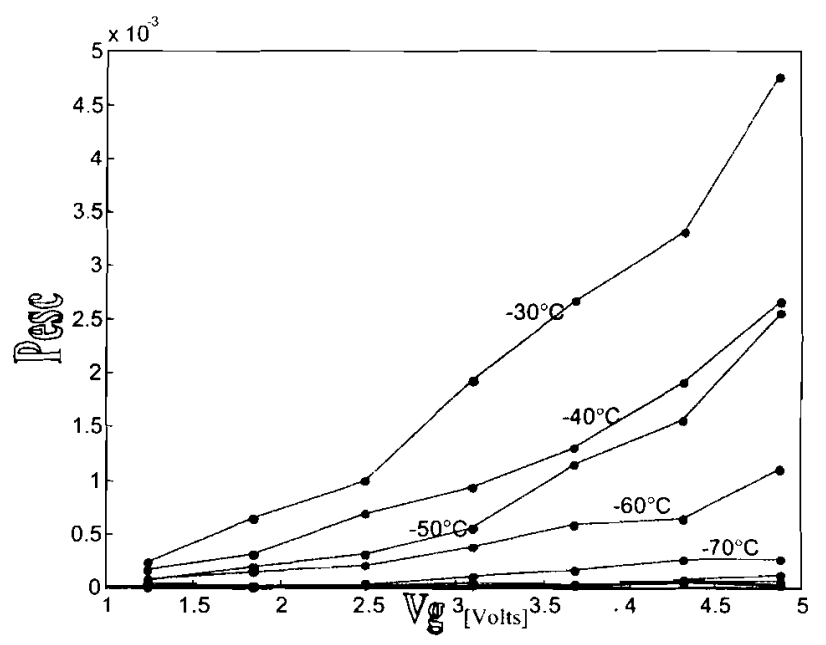

Figura 12. Probabilidade de contagem de escuro versus amplitude do pulso de gatilho para 9 diferentes temperaturas.

$\mathrm{O}$ resultado experimental da eficiência quântica pode ser observado na Figura 13, para valores de amplitude do pulso de gatilho $\left(V_{g}\right)$ no intervalo $[1 \mathrm{~V}, 5 \mathrm{~V}]$ e temperaturas no intervalo $\left[-150^{\circ} \mathrm{C},-30^{\circ} \mathrm{C}\right]$.

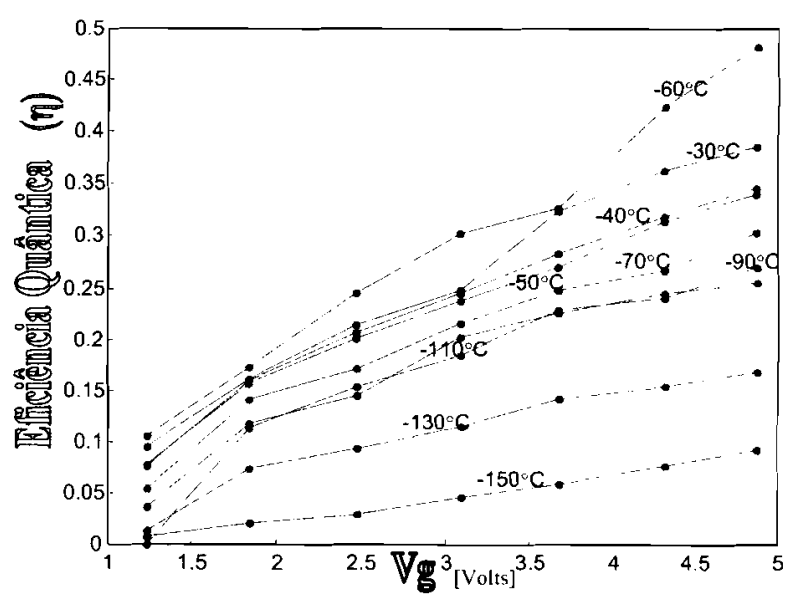

Figura 13. Eficiência quântica versus amplitude do pulso de gatilho para 9 diferentes temperaturas.

Com exceção da curva para $-60^{\circ} \mathrm{C}$, que estranhamente apresentou excelente resultado, observamos no geral que, quanto maiores forem a temperatura e amplitude de $V_{g}$ maior a eficiência quântica. A discrepância para a curva de $-60^{\circ} \mathrm{C}$ pode ter sido causada por algum fator de ruído como, 
por exemplo, a momentânea absorção de luz ambiente pelo FDA. Em sistemas de DQC usando interferômetros com máxima visibilidade a taxa de bits quânticos errados, QBER, é dada por

$$
Q B E R=\frac{P_{e s c}}{2 P_{e s c}+e^{-\alpha L} \eta\langle n\rangle},
$$

sendo $\alpha$ o coeficiente de perdas da fibra e $L$ o comprimento de fibra entre transmissor e receptor. Para o sistema ser seguro a QBER deve ser menor que $15 \%[1,2,7,8]$. Para $\langle n\rangle=0,1, \alpha=0,2 \mathrm{~dB} / \mathrm{km}$ e $L=40 \mathrm{~km}, \mathrm{P}_{\mathrm{esc}}=2 \times 10^{-4}$ e $\eta=0,2$ obtemos QBER $=5,6 \%$. Observando as Figuras 12 e 13, vemos que aqueles valores de $P_{e s c}$ e $\eta$ podem ser obtidos operando o FDA testado em, aproximadamente, $-70^{\circ} \mathrm{C}$ e $V_{g}$ $=3 \mathrm{~V}$. Por fim, na Figura 14 temos os pulsos de gatilho e de saída do circuito de extinção $\left(V_{s}\right)$, devido à uma contagem de escuro (ruído), na temperatura ambiente. Os parâmetros são: $V_{F D A}=49 \mathrm{~V}, V_{g}=3,30 \mathrm{~V}, T_{h}=8 \mathrm{~ns}, R_{\Gamma}=330 \mathrm{k} \Omega, C_{s}=100 \mathrm{nF}$, com $V_{b}=50 \mathrm{~V}$. No pulso devido à avalanche, vemos a ação do integrador (overshooting negativo) quando da descida do pulso de gatilho.

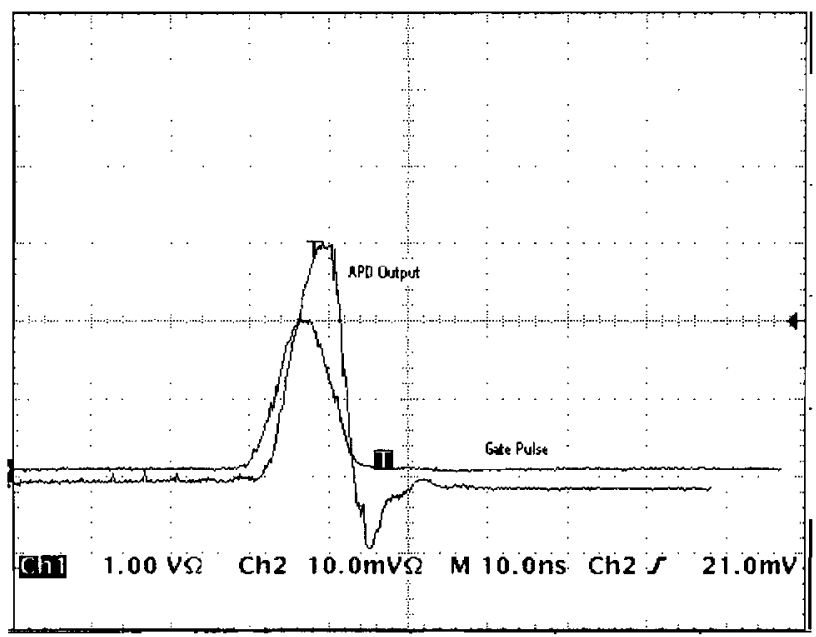

Figura 14. Pulsos de gatilho e avalanche vistos em um osciloscópio.

\section{CONCLUSÃO}

Apresentamos as condições iniciais para a montagem de um sistema de detecção de fótons isolados, na janela de $1550 \mathrm{~nm}$, para uso em sistemas de distribuição quântica de chaves. A temperatura de operação e a tensão de excesso devem ser cuidadosamente escolhidas para otimizar o funcionamento do FDA. Para este fim, determinamos as curvas experimentais da probabilidade de contagem de escuro e da eficiência quântica de um FDA. Nestas curvas podemos determinar um ponto de operação no qual o sistema de DQC tenha uma QBER menor que o limite de segurança de $15 \%$. O circuito de extinção de avalanche gatilhado também foi analisado através de simulações numéricas. Nas simulações apresentadas podemos perceber que, a situação na qual a extinção ocorre no fim do pulso de gatilho é melhor do que a situação na qual a extinção ocorre durante o pulso de gatilho, pois a tensão sobre o FDA é maior, o pulso de avalanche é mais facilmente detectado em relação aos picos na borda do pulso de gatilho e uma maior taxa de transmissão é possível.

\section{AGRADECIMENTOS}

Este trabalho foi financiado pela CAPES e CNPq. Os autores são gratos a Phil Marsden e Daniel Ljunggren, IMIT/KTH-Suécia, pela colaboração nas medidas experimentais realizadas.

\section{REFERÊNCIAS}

[1] Simon J. D. Phoenix and Paul D. Townsend, "Quantum cryptography: how to beat the code breakers using quantum mechanics", Contemporary Physics, vol. 36, p. 165-195, 1995.

[2] N. Gisin, G. Ribordy, W. Tittel and H. Zbinden, "Quantum cryptography", quant-ph/0101098, http://xxx.lanl.gov, 1998

[3] W. K. Wootters and W. H. Zurek, "A single quantum cannot be cloned", Nature, no. 299, p. 802-803, 1982

[4] S. Cova, M. Ghioni, A. Lacaita, C. Samori and F. Zappa, "Avalanche photodiodes and quenching circuits for singlephoton detection", Applied Optics, vol. 35, no. 129, p. 1956$1976,1996$.

[5] A. Lacaita, F. Zappa, S. Cova, and P. Lovati, "Single-photon detection beyond $1 \mathrm{\mu m}$ : Performance of commercially available InGaAs/InP detectors", Applied Optics, vol. 35, no. 16, p. 2986-2996, 1996.

[6] G. Ribordy, J. D. Gautier, H. Z., and N. Gisin, "Performance of InGaAs/InP avalanche photodiodes as gated-mode photon counters", Applied Optics, vol. 37, no. 12, p. 2272-2277, 1998.

[7] F. Gibson, Experimental evaluation of Quantum Cryptography system for $1550 \mathrm{~nm}$, Master of Science thesis, Department of eletronics-QEO, Kungl Tekniska Högskolan, 1998.

[8] M. Bourennane, D. Ljunggren, A. Karlsson, P. Jonsson, A. Hening and J. P. Ciscar, "Experimental long wavelength quantum cryptography: from single-photon transmission to key extraction protocols", Journal of Modern Optics, vol. 47, no. $2 / 3$, p. $563-579,2000$

George André Pereira Thé é graduando em Engenharia Elétrica na Universidade Federal do Ceará.

Rubens Viana Ramos graduou-se em Engenharia Elétrica em 1996 na UFC, obteve os graus de Mestre e Doutor em Engenharia Elétrica, na UNICAMP, em 1998 e 2000, respectivamente. Desde 2002 é pesquisador associado ao DETI/UFC. 